\title{
Chemical Peel
}

National Cancer Institute

\section{Source}

National Cancer Institute. Chemical Peel. NCI Thesaurus. Code C93203.

A dermatologic procedure that uses a chemical solution to remove the outer epidermal

layers in an effort to smooth the texture of the skin. 